\section{Multiple Target Detection Using Modified High Order Correlations}

\author{
REN-JEAN LIOU, Member, IEEE \\ MAHMOOD R. AZIMI-SADJADI, Senior Member, IEEE \\ Colorado State University
}

This work is concerned with the problem of multiple target track detection in heavy clutter. Using the "modified high order correlation" (HOC) [1-4] process and a track scoring mechanism a new method is developed to perform data association and track identification in the presence of heavy clutter. Using this new scheme any number of very close, crossing or splitting target tracks can be resolved without increasing the computational complexity of the algorithm. The applicability of the method for continuous detection of target tracks that can originate and terminate at any scan is also demonstrated. In addition, the operating characteristics as a function of the clutter density are also provided. Simulation results on all the cases are presented.

Manuscript received July 15, 1996; revised April 15, 1997.

IEEE Log No. T-AES/34/2/03199.

This work was supported in part by IBM Corporation, Federal Sector Division, Boulder, CO 80301.

Authors' current addresses: R.-L. Liou, Dept. of Electrical Engineering, Da-Yeh Institute of Technology, Changhua, Taiwan, ROC; M. R. Azimi-Sadjadi, Dept. of Electrical Engineering, Colorado State University, Fort Collins, CO 80523.

$\overline{0018-9251 / 98 / \$ 10.00 ~(C) ~} 1998$ IEEE

\section{INTRODUCTION}

The problem of detection and identification of multiple low observable targets in a heavily cluttered background is the primary focus of this work. The advances made in algorithm and hardware developments have increased the processing capabilities of the detection schemes for operation in more difficult environments. However, because of the complicated nature of the problem, no general solution is available for all cases. Some contributing factors for this deficiency are: extremely low signal-to-noise/clutter ratio, nonrepeatability of the target signature, competing clutter forming returns with similar shapes as those of the actual targets, obscured targets and lack of a priori information about the initial conditions and the signal statistics. In addition, the presence of multiple targets complicates the detection, data association and classification processes, especially when targets are allowed to cross, split, remain close together or be partially hidden in the background.

Over the years, several target detection techniques have been proposed in the literature [5-15] which use different schemes such as spatio-temporal filtering $[5,6]$, maximum likelihood (ML) estimation [7, 14], recursive Kalman filtering [8-13] and neural networks [15]. In [6] a 3-D spatial-temporal filtering scheme is developed. The operations are done in the frequency domain by passing the image through a bank of directional filters each tuned to extract line features of the tracks with certain orientations. Recently, Bar-Shalom, et al. [11, 12] used two different centroid-based measurements to track targets in forward looking infrared (IR) data. These provide information about the location of the single-frame centroid and the offset of the centroid measured based on two consective frames. The state variable models and the corresponding Kalman filters were then derived in which the target centroid is estimated based upon these measurements. Comparison between the two filters indicated that the filter which used autocorrelated measurement noise provided better performance. In a more recent paper [13] Shertukde and Bar-Shalom extended this approach by using the joint probablistic data association (JPDA) in conjunction with a Kalman state estimator. The "merged measurement" model in the state equations presents a linear combination of centroids of an individual target. This method is useful for tracking crossing targets. The scheme in [14] uses a track-before-detect procedure to detect multiple targets in presence of false measurements. The methodology is based upon ML estimation as the measurement model is nonlinear. Roth [15] developed a neural network-based scheme for detection of straight line tracks in the background clutter. A Hopfield network was trained to implement the 
optimum post-detection target track receiver operation.

In all of the above-mentioned schemes certain assumptions about the target signatures and the background clutter are made in order to reduce the computational requirements. For applications where such assumptions are valid, these techniques perform well. However, if no a priori information about the statistics of signals and clutter and/or noise is available and further the signals are not simply distributed or are highly correlated, these techniques may give inferior results.

In [1-4], a new method referred to as high order correlation (HOC) was developed which does not make any a priori assumption about the targets and background clutter. This method was modified by imposing velocity and curvature constraints in order to reject false tracks even at a greater degree and improve clutter rejection performance. Both the original and modified HOC methods exploit the temporal and spatial dependencies of consecutive data points on a target track to discriminate them from background clutter. The real-time implementation of these methods using connectionist networks was also presented in [1-4] which showed the potential of these schemes for parallel implementation. The algorithm was tested on numerous target events and an average clutter rejection rate of $98 \%$ under moderate clutter density was achieved without losing any valid information pertaining to the target tracks. The comparison of the results with those of the 3-D spatio-temporal filtering [6] indicated the superiority of the modified HOC method.

This work extends the applicability of the modified HOC method to practical scenarios which involve detection of multiple targets in heavy clutter. This problem is complicated especially when the targets are allowed to be crossing, splitting, or very close together. The applicability of the modified HOC method for multiple target detection scenarios under various clutter densities is demonstrated without increasing the complexity of the algorithm. In addition, it is shown that the detection can be performed without any prior knowledge of the number of targets, the target's dynamical information and initial conditions.

A new scoring process is presented which can be used in conjunction with the modified HOC scheme to achieve better clutter rejection performance. Scoring is a mechanism which assigns likelihood measures to the spatio-temporal sequences in order to determine their resemblance to real target tracks. We demonstrate here how one can easily extract score information during the modified HOC process without adding computational overhead to the alogorithm. Substantial improvements in clutter rejection can be achieved by rejecting the sequences with low scores.
In order to better identify multiple targets individually, the properties of the modified HOC method are exploited to perform data point association in consecutive scans, formulate independent target tracks and reject the adjacent clutter. The results not only indicated significantly better target identification and clutter rejection rate but also showed the potential of this approach for other applications such as track classification, tracking, and data fusion.

In a real target detection environment, there is usually no a priori knowledge as to when the targets initiate and terminate in the field of view. To detect a target in such an environment, the sensor must operate continuously and be capable of detecting the presence of the target at any number of scans as soon as there is sufficient information for making the decision. Simulation results in this work also demonstrate the ability of the HOC method to perform continuous target detection and clutter rejection. This is achieved with only little modifications to the algorithm.

Experiments were also conducted on various processing conditions where the clutter density and the order of $\mathrm{HOC}$ are variable. The upper bound on the clutter density using this method is also analyzed. The purpose is to study how to fully utilize the benefits of the modified HOC scheme so that the detection can be made in a minimum number of scans using an optimum structure.

This paper is organized as follows. The modified HOC scheme and its important properties are first established in Section II. Section III presents the scoring and track identification processes using the information available directly from the modified HOC. Simulation results are presented in Section IV for various practical scenarios discussed above. Finally, conclusions are given in Section V.

\section{SPATIO-TEMPORAL HOC METHOD}

A multiscan image is actually obtained by stacking several 2-D images collected at some time intervals by an IR sensor whose field of view is fixed with respect to the background. This yields a 3-D image in the Cartesian coordinates $(x, y, t)$ where $(x, y)$ are the spatial variables and $t$ represents the time. A moving target in the sensed scene forms a track or a signature in this 3-D image.

In the IR imagery the intensity of the target is usually unknown and can vary substantially from one scan to the next depending on the temperature, sun angle, etc. As a result, target detection using intensity-based schemes, such as thresholding, naturally leads to an unacceptable performance. To retain dim targets (dimmer than background), in this work the scan data is converted to binary images by mapping any sensor return with intensity greater than zero to one (1). This process yields a 2-D image for 
every scan data in which each pixel at $(x, y)$ location is either "blank", if: there is no sensor return at that location, or one (1), if there is a return associated with target or clutter. Now, stacking several such binary images for different time scans gives a 3-D image which can be modeled [1] by

$$
F\left(x, y, t_{n}\right)=S\left(x, y, t_{n}\right)+\left[1-S\left(x, y, t_{n}\right)\right] N\left(x, y, t_{n}\right)
$$

where $x, y$ represent the spatial locations on a discrete $2-\mathrm{D}$ plane, $t_{n}$ is the time unit or scan number, and $F(\cdot)$ is the observed binary image consisting of two disjoint parts, i.e., target image $S(\cdot)$, and clutter image $N(\cdot)$, which are also binary. This model merely states that the observed image is an additive combination of disjoint images associated with the target and clutter and that a particular point in the 3-D image corresponds to either target or clutter but the two do not occur at the same location and time.

Since a target moves in a certain direction and builds a time-dependent track in the 3-D space, there exists spatio-temporal correlations between consecutive target points. To find this dependency, we can use the following equation [1]

$$
Y\left(x, y, t_{n}\right)=g\left[\sum_{i=-|v|}^{|v|} \sum_{j=-|v|}^{|v|} F\left(x, y, t_{n}\right) F\left(x+i, y+j, t_{n+1}\right)\right]
$$

where $v$ is the maximum allowable target movement from scan to scan and $Y(\cdot)$ represents how the $(x, y)$ points of $F(")$ at scan $t_{n}$ are correlated to their neighboring points, within a window of size $(2 v+$ 1) $X(2 v+1)$, at $\operatorname{scan} t_{n+1}$. The function $g(\cdot)$ is a hard limiter threshold function defined as

$$
g(c)= \begin{cases}1, & \text { if } \quad c>0 \\ 0, & \text { if } \quad c \leq 0\end{cases}
$$

Obviously, if $Y=1$, then there is a two-point spatio-temporal sequence initiated at location $(x, y)$ and at scan $t_{n}$ to location $(x+i, y+j)$ at scan $t_{n+1}$. Although this process can be repeated to identify all such two-point sequences, it cannot provide correlation information of more than two data points.

To determine the spatio-temporal correlations of more than two data points, we build memory into the process and compute the higher order correlations of $Y$ by using a recursive procedure [1] with the general form of

$$
\begin{aligned}
Y^{(k)}\left(x, y, t_{n}\right)=g[ & \sum_{i=-|v|}^{|v|} \sum_{j=-|v|}^{|v|} Y^{(k-1)}\left(x, y, t_{n}\right) \\
& \left.\times Y^{(k-1)}\left(x+i, y+j, t_{n+1}\right)\right]
\end{aligned}
$$

where $n$ can vary from 1 to $n_{\max }$, and $k$ is the order of the recursion. When the order $k$ increases $n_{\max }$ should decrease such that at all times $k+n_{\max }=n_{0}$ where $n_{0}$ is number of scans used for detection [1]. Note that for $k=1, Y^{(0)}\left(x, y, t_{n}\right)=F\left(x, y, t_{n}\right)$ and hence $Y^{(1)}\left(x, y, t_{n}\right)$ provides 2-point correlation as in (2). For $k=2, Y^{(2)}\left(x, y, t_{n}\right)$ gives the correlation of $Y^{(1)}\left(x, y, t_{n}\right)$ and $Y^{(1)}\left(x, y, t_{n+1}\right)$, thus providing a 3-point correlation. Consequently, $Y^{(k)}\left(x, y, t_{n}\right)$ gives correlational information among $k$ consecutive scans, i.e., if $Y^{(k)}\left(x, y, t_{n}\right)=1$, then there may exist a track extending from location $\left(x, y, t_{n}\right)$ at scan $t_{n}$ to a point at scan $t_{n+k}$; otherwise these data points do not lie on a possible track and can subsequently be removed. The processed result of the HOC process in (4) is a 3-D image in which all the points satisfy the spatio-temporal correlational criterion and the background clutter is substantially removed.

In [1], it was also shown that the entire process can be implemented on a connectionist network consisting of several planar layers of neurons with hard limiter activation functions. The number of layers to be used depends on the choice of the order for the process. The output of the final layer provides the HOC image result.

The limitation of the HOC process, however, lies in the fact that beside the maximum target movement, this process does not impose any other constraints based on the target dynamical information such as curvature and velocity variations. As a result, certain desirable clutter rejection rates may not be achieved as clutter with returns similar in shape to a jagged track would also satisfy the HOC criterion and hence will be retained in the final image. The problem can be remedied by modifying the HOC process in (4) to include spatio-temporal correlations in three consecutive scans. In this "modified HOC" the possible movement from scan $t_{n}$ to scan $t_{n+1}$ can be used to determine the movements to the $t_{n+2}$ scan. This can be portrayed by using the following equation [1]

$$
\begin{aligned}
Y^{(k)}\left(x, y, t_{n}\right)=g & {\left[Y^{(k-1)}\left(x, y, t_{n}\right) \sum_{i_{1}} \sum_{j_{1}} Y^{(k-1)}\left(x+i_{1}, y+j_{1}, t_{n+1}\right)\right.} \\
& \left.\times \sum_{i_{2}} \sum_{j_{2}} Y^{(k-1)}\left(x+i_{1}+i_{2}, y+j_{1}+j_{2}, t_{n+2}\right)\right]
\end{aligned}
$$

where $\left(i_{1}, j_{1}\right)$ and $\left(i_{2}, j_{2}\right)$ represent the possible movements from scans $t_{n}$ to $t_{n+1}$ and $t_{n+1}$ to $t_{n+2}$, respectively. In (5), to compute the correlations between scans $t_{n}$ and $t_{n+1}$, we considered all the possible movements and used the entire velocity window, i.e., $i_{1}, j_{1} \in[-|v|,|v|]$. However, for each pair of $\left(i_{1}, j_{1}\right)$ generally only a subset of $\left(i_{2}, j_{2}\right)$ values can be acceptable. This subset which determines the size and shape of the velocity window between $t_{n+1}$ and $t_{n+2}$ is obtained based upon a given maximum moving 

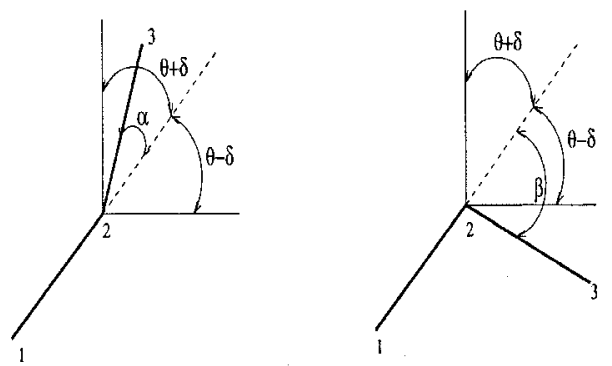

Fig. 1. Two 3-point tracks. Left one satisfies modified HOC criterion while right one does not.

curvature constraint [1]. To see this, let us consider the target bearing angle $\theta$ from scan $t_{n}$ to $t_{n+1}$ which can be calculated using

$$
\theta=\tan ^{-1} \frac{j_{1}}{\dot{i}_{1}}
$$

Now, if the maximum target moving curvature is $\delta$ then the turning angle from scan $t_{n+1}$ to $t_{n+2}$ should be within a bounded region, i.e.,

$$
\theta-\delta \leq \tan ^{-1} \frac{j_{2}}{i_{2}} \leq \theta+\delta
$$

Having specified the variables $\theta$ and $\delta$, the acceptable range of the values of $\left(i_{2}, j_{2}\right)$ in (5) can be determined using (6). Thus, the range of movements from scan $t_{n+1}$ to $t_{n+2}$ is limited according to the assumed maximum moving curvature. Since there are limited cases of $\left(i_{1}, j_{1}\right)$, the computation in (6) can simply be replaced by a look-up table as shown in [1].

To see the advantages of the modified HOC in comparison with the original HOC, let us consider the simple example in Fig. 1 which shows two 3-point tracks in the $x y$ plane moving from point 1 to 3 . The moving angle from point 1 to 2 is $\theta$ and the angle difference from point 1 to 2 and that of point 2 to 3 is $\alpha$ for the track on the left and $\beta$ for the one on the right. From (4), both of these tracks satisfy the condition in the original HOC method which does not impose any constraint. If we choose the maximum moving curvature constraint of $\delta$, obviously the track on the left falls within the range $[\theta-\delta, \theta+\delta]$ in (6) while the track on the right does not. Therefore, this track would not satisfy the curvature constraint of the modified HOC criterion and hence will be rejected.

It was shown [1] that the original HOC method can provide correlational information for $k+1$ consecutive scans, where $k$ is the order of the HOC. The following result shows that the modified HOC of the same order provides spatio-temporal correlational information for $2 k+1$ scans, i.e., almost twice that of its original counterpart.

PROPOSITION 1 Assume that target points are located at $\left(x_{n}, y_{n}\right)$ for each corresponding scan $t_{n}$. After

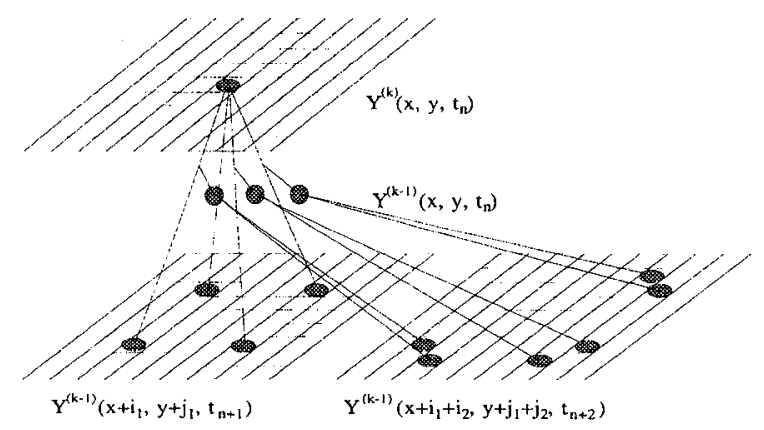

Fig. 2. Connectionist network for one layer of modified HOC.

computing the modified HOC's recursively, if the kth order correlation $Y^{(k)}\left(x_{n}, y_{n}, t_{n}\right)=1$, then there exists a track that consists of $2 k+1$ points extending from scan $t_{n}$ to $t_{n+2 k}$, i.e.,

$$
\begin{aligned}
Y^{(k)}\left(x_{n}, y_{n}, t_{n}\right)= & F\left(x_{n}, y_{n}, t_{n}\right) F\left(x_{n+1}, y_{n+1}, t_{n+1}\right) \\
& \times \cdots F\left(x_{n+2 k}, y_{n+2 k}, t_{n+2 k}\right)=1 .
\end{aligned}
$$

\section{Proof See Appendix A.}

In [1], a neural network implementation of (5) was suggested using high order neurons $[13,14]$. The structure of a typical layer for computing first-order modified HOC is illustrated in Fig. 2. Multiple layers can be cascaded to compute modified HOC of higher order. The output of the final layer provides the clutter-free processed image. Simulation results in [1] on various target events indicated an average clutter rejection rate of $98 \%$ for moderately dense clutter (density less than $0.8 \%$ per scan). ${ }^{1}$ This close to perfect clutter rejection rate of the modified HOC is achieved without perturbing or losing the target information. However, for events with clutter density higher than $1.1 \%$, more false tracks were left in the final image. These false tracks satisfy the spatio-temporal criterion of the modified HOC process. It is shown later that most of these false tracks can be removed by incorporating a scoring scheme into the modified HOC process.

\section{MULTIPLE TRACK IDENTIFICATION PROCESS}

Conventional target tracking approaches which rely on data association process [8-10] become computationally intensive in dealing with multiple target problems, especially when tracks are very close and allowed to cross or split. These methods perform track-before-detect procedure and thus are very complicated for detection only purposes. The modified HOC method provides a straightforward and an easy to implement scheme for detecting the

\footnotetext{
${ }^{1}$ The clutter density is measured by the percentage of random noise pixels out of all possible pixels in one scan.
} 


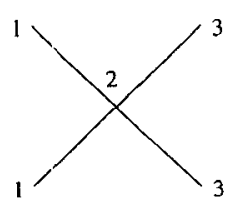

(a)

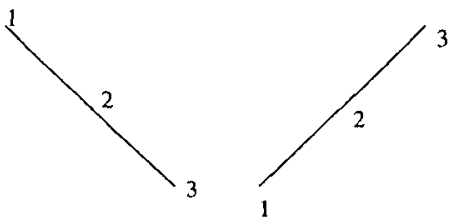

(b)

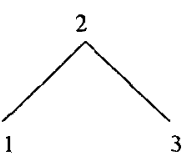

(d)

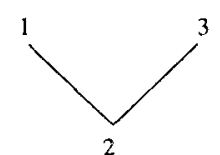

(e)

Fig. 3. (a) Crossing tracks. (b)-(e) Possible individual tracks in (a).

presence of multiple targets before identifying each individual track in the scene.

If $n_{0}$ scans are to be used for detection, using (5) and the results in Proposition 1, it can be shown that any track that extends over more than $2 k+1$ scans, where $2 k+1<n_{0}$, will meet the modified $\mathrm{HOC}$ criterion and hence will be retained in the processed image. This is achieved regardless of the number and locations of the targets in the scene and their dynamical behavior. Neverthelees, the clarity in identifying indiviclual tracks obviously depends upon the density of the surrounding background clutter and the closeness of tracks to each other. Some clutter points which are remained in the processed images and are near the real tracks may cause ambiguity in identifying the individual tracks. In this section new schemes for scoring and track identification are introduced in order to improve the capability of the modified HOC in identifying individual tracks in a multitarget scenario.

\section{A. Scoring Process}

Significant improvements in the performance can be obtained by incorporating a scoring process in the modified HOC method. Scoring information can be extracted rather easily from the modified HOC process and then be used to associate target points and determine the resemblance of a sequence of points to a real target track. In this section, we discuss how the score information can be extracted for each possible target point and then develop an efficient method to identify individual target tracks in a scene.

One of the most popular scoring process is the probabilistic data association [8-10] for multitarget tracking problems. It provides measurements on different sensor returns to determine if they have a common source. To obtain the scores soft decision is typically used. This permits the data to be assigned to multiple sets with each candidate having a measure of confidence. The problem of data association is highly complicated, since the number of possible hypothesis associated with different returns increases rapidly with the number of returns. The presence of clutter further increases this complexity.

Data association can be performed directly using the modified HOC process based upon its inherent properties. Using (5), it is possible to arrive at a movement and a curvature dependent scoring criterion based upon values of $\left(i_{1}, j_{1}\right)$ and $\left(i_{2}, j_{2}\right)$. The movements between $t_{n}, t_{n+1}$ and $t_{n+1}, t_{n+2}$ are $\left|i_{2}-i_{1}\right|$ and $\left|j_{2}-j_{1}\right|$ in $x$ and $y$ directions, respectively. Since $i_{1}, i_{2}, j_{1}, j_{2} \in[-v, v]$, where $v$ is the maximum target movement, thus $0 \leq\left|i_{2}-i_{1}\right| \leq 2 v$ and $0 \leq\left|j_{2}-j_{1}\right| \leq$ $2 v$. For real target tracks, the scan-to-scan variations of movements $\left|i_{2}-i_{1}\right|,\left|j_{2}-j_{1}\right|$ and curvature $\left|\tan ^{-1}\left(j_{2} / i_{2}\right)-\tan ^{-1}\left(j_{1} / i_{1}\right)\right|$ are typically small.

Two kinds of scores,based upon the movement and the curvature, can be derived using $\left(i_{1}, j_{1}\right)$ and $\left(i_{2}, j_{2}\right)$ values. The scoring based upon the movement can be generated using

$$
\text { score }_{\text {move }}=\left(A_{1}-\left|i_{2}-i_{1}\right|-\left|j_{2}-j_{1}\right|\right) \times \alpha_{1}
$$

where $A_{1}$ is a constant dependent on the maximum velocity $v$ and $\alpha_{1}$ is a scaling factor which is empirically determined. In this scoring, the highest score is obviously $A_{1} \times \alpha_{1}$ which occurs when $i_{1}=i_{2}$ and $j_{1}=j_{2}$.

The curvature information can easily be obtained from the look up table of $\left(i_{2}, j_{2}\right)$ values (see [1]). Thus, the curvature score can be computed using

$$
\text { score }_{\text {angle }}=\left(A_{2}-\Gamma\right) \times \alpha_{2}
$$

where

$$
\Gamma=\left|\tan ^{-1} \frac{j_{2}}{i_{2}}-\tan ^{-1} \frac{j_{1}}{i_{1}}\right| \leq \delta
$$

is the difference between the scan-to-scan angles. Again, $A_{2}$ and $\alpha_{2}$ are parameters similar to those in (8). These scores can be obtained directly during the modified HOC process.

The score information is of particular importance when several possible tracks are close together. A simple example is demonstrated in Fig. 3(a) in which there are two target tracks extending over three consecutive scans. The associated scan number for each target point is indicated. Since no information regarding the target movement or direction is available, two possible pairs of tracks can be identified. Figs. 3(b) and 3(c) show the first pair where the tracks are linear while Figs. 3(d) and 3(e) show two tracks with sharp turns in the middle. The corresponding movements for the three scans for each possible track in Fig. 3 are given in Table I. Let $A_{1}=9, \alpha_{1}=0.5, A_{2}=2.356$, and $\alpha_{2}=1.91$, then using (8) and (9) it is realized that the highest possible 
TABLE I

Individual Target Movements and Associated Scores

\begin{tabular}{|c|c|c|c|c|}
\hline Track & $\left(i_{1}, j_{1}\right)$ & $\left(i_{2}, j_{2}\right)$ & Velocity Score & Curvature Score \\
\hline b & $(1,1)$ & $(1,1)$ & 4.5 & 4.5 \\
\hline c & $(1,-1)$ & $(1,-1)$ & 4.5 & 4.5 \\
\hline d & $(1,1)$ & $(1,-1)$ & 3.5 & 1.5 \\
\hline e & $(1,-1)$ & $(1,1)$ & 3.5 & 1.5 \\
\hline
\end{tabular}

movement and curvature scores is 4.5 . The associated scores for each track are calculated using (8) and (9). These are provided in Table I. It is obvious that targets with more consistent dynamical motion produce higher scores. Consequently, when several tracks are crossing, splitting, or close together, the score information provides a measure to identify the most likely tracks. However, when clutter points are close to the tracks they may also satisfy the modified HOC process hence increasing the total number of hypothesis. Even though most of these points can easily be removed as they generally lead to low scores, it is desirable to reduce the total number of possible tracks using a data association process.

Additionally, the above scoring process applies to each individual point. The determination of the resemblance of a sequence to a real track is achieved by taking the average of the point scores on the sequence. However, this can only be made possible when all the possible target tracks are identified. In the following section a new track identification process is introduced which can be used to perform data association and determine track evolution and also facilitate the scoring process of individual tracks.

\section{B. Track Identification Process}

In the resultant image obtained using the modified HOC, $Y^{(k)}\left(x, y, t_{n}\right)=1$ indicates that the sets of $\left(i_{1}, j_{1}\right)$ and $\left(i_{2}, j_{2}\right)$ form a three-point track for three consecutive scans $t_{n}, t_{n+1}$ and $t_{n+2}$. This "short track" may or may not be part of a complete target track. If a complete target track exists, these three-point sequences consistently occur in the modified HOC image for all the scans in which case it is possible to define the entire track by associating these points. However, at this time there is no effective way of performing this association, since using serial processing often requires exhaustive search especially for a densely cluttered environment.

An efficient method can be developed by combining the forward and backward correlations in the modified HOC in order to provide five-point track information directly. The backward correlations are evaluated using

$$
\begin{array}{r}
Y^{(k)}\left(x, y, t_{n}\right)=g\left[Y^{(k-1)}\left(x, y, t_{n}\right) \sum_{i_{1}} \sum_{j_{1}} Y^{(k-1)}\left(x+i_{1}, y+j_{1}, t_{n-1}\right)\right. \\
\left.\quad \times \sum_{i_{2}} \sum_{j_{2}} Y^{(k-1)}\left(x+i_{1}+i_{2}, y+j_{1}+j_{2}, t_{n-2}\right)\right] .
\end{array}
$$

To demonstrate this idea let us consider a second-order modified HOC for ten scans of data. In the forward process, the HOC images can be obtained from scans $t_{1}$ to $t_{6}$ (see Proposition 1). On the other hand, the backward process would provide images from scans $t_{10}$ to $t_{5}$. From the results in scan 5 , the data points in scans 3-7 can be associated, i.e., a five-point sequence $\left(x+i_{1 b}+i_{2 b}, y+j_{1 b}+j_{2 b}, t_{3}\right)$, $\left(x+i_{1 b}, y+j_{1 b}, t_{4}\right),\left(x, y, t_{5}\right),\left(x+i_{1 f}, y+j_{1 f}, t_{6}\right)$, and $\left(x+i_{1 f}+i_{2 f}, y+j_{1 f}+j_{2 f}, t_{7}\right)$ is created where the subscripts $b$ and $f$ represent backward and forward processes, respectively. Similarly, from the results in scan 6, another five-point sequence for scans $4-8$ can be obtained. Thus, by comparing the results at scans 5 and 6 , an eight-point sequence may be identified instantly. This method considerably simplifies the effort for finding a complete track in ten scans.

Although the above method provides a quick way to formulate a track, the points $\left(x+i_{1 b}+i_{2 b}\right.$, $\left.y+j_{1 b}+j_{2 b}, t_{n-2}\right),\left(x+i_{1 b}, y+j_{1 b}, t_{n-1}\right),\left(x, y, t_{n}\right)$, $\left(x+i_{1 f}, y+j_{1 f}, t_{n+1}\right)$, and $\left(x+i_{1 f}+i_{2 f}\right.$, $\left.y+j_{1 f}+j_{2 f}, t_{n+2}\right)$ may not satisfy the criterion for the modified HOC and thus may be eliminated from the resultant image. Some interesting observations can be made by investigating the occurrence of each point. Owing to the similarity of the forward and backward correlation processes, only the example for forward correlation is given below.

Suppose that there are three points located at $\left(x_{n}, y_{n}, t_{n}\right),\left(x_{n+1}, y_{n+1}, t_{n+1}\right)$, and $\left(x_{n+2}, y_{n+2}, t_{n+2}\right)$ for three consecutive scans and that $\left(i_{1}, j_{1}\right)$ and $\left(i_{2}, j_{2}\right)$ are associated with $\left(x_{n}, y_{n}, t_{n}\right)$, i.e., $x_{n+1}=$ $x_{n}+i_{1}, y_{n+1}=y_{n}+j_{1}$ and $x_{n+2}=x_{n}+i_{1}+i_{2}$, $y_{n+2}=y_{n}+j_{1}+j_{2}$, where $\left(i_{1}, j_{1}\right)$ and $\left(i_{2}, j_{2}\right)$ are obtained during the modified HOC process when $Y^{(k)}\left(x_{n}, y_{n}, t_{n}\right)=1$. However, this does not necessarily imply that $\left(x_{n}+i_{1}, y_{n}+j_{1}, t_{n+1}\right)$ or $\left(x_{n}+i_{1}+i_{2}, y_{n}+\right.$ $j_{1}+j_{2}, t_{n+2}$ ) occur in the modified HOC image, i.e., $Y^{(k)}\left(x_{n+1}, y_{n+1}, t_{n+1}\right)=1$ or $Y^{(k)}\left(x_{n+2}, y_{n+2}, t_{n+2}\right)=1$. Different scenarios can be observed on the occurrence of $Y^{(k)}\left(x_{n+1}, y_{n+1}, t_{n+1}\right)$ and $Y^{(k)}\left(x_{n+2}, y_{n+2}, t_{n+2}\right)$ in the resultant image. Typical examples of these scenarios are depicted in Fig. 4.

Case 1 When $Y^{(k)}\left(x_{n+1}, y_{n+1}, t_{n+1}\right)=1$ and $Y^{(k)}\left(x_{n+2}, y_{n+2}, t_{n+2}\right)=1$, a consistent correlation for five consecutive scans is obtained which implies that there is a five-point sequence from $t_{n}$ to $t_{n+4}$ in the original image (see Fig. 4(a)). 


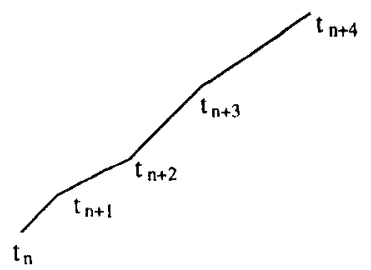

(a)

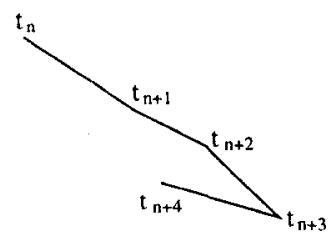

(c)

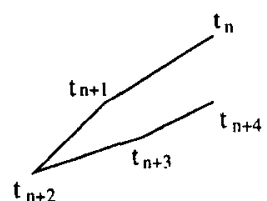

(b)

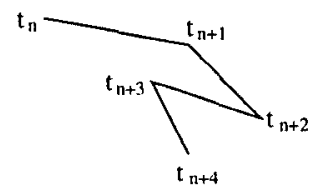

(d)

Fig. 4. Different sceriarios for occurrence of $Y^{(k)}\left(x_{n+1}, y_{n+1}, t_{n+1}\right) Y^{(k)}\left(x_{n+2}, y_{n+2}, t_{n+2}\right)$. (a) Complete track for 5 scans. (b) Sharp change at $t_{n+2}$. (c) Sharp change at $t_{n+3}$. (d) Sharp change at $t_{n+2}$ and $t_{n+3}$.

Case 2 When $Y^{(k)}\left(x_{n+1}, y_{n+1}, t_{n+1}\right)=0$ and $Y^{(k)}\left(x_{n+2}, y_{n+2}, t_{n+2}\right)=1$, it implies that the movements from scans $t_{n+1}, t_{n+2}$ to $t_{n+3}$ in the original image do not satisfy the criterion in the modified HOC scheme. This can be caused by a sharp curvature change in the movements between $t_{n+1}$ to $t_{n+2}$ and $t_{n+2}$ to $t_{n+3}$ (see Fig. 4(b)).

Case 3 When $Y^{(k)}\left(x_{n+1}, y_{n+1}, t_{n+1}\right)=1$ and $Y^{(k)}\left(x_{n+2}, y_{n+2}, t_{n+2}\right)=0$, two possible cases could occur. a) The movements between scans $t_{n+2}, t_{n+3}$ and $t_{n+4}$ do not satisfy the modified HOC criterion which may occur due to a sharp turning point at scan $t_{n+3}$ (see Fig. 4(c)). b) The target point at scan $t_{n+4}$ is misdetected by the sensor.

Case 4 When $Y^{(k)}\left(x_{n+1}, y_{n+1}, t_{n+1}\right)=0$ and $Y^{(k)}\left(x_{n+2}, y_{n+2}, t_{n+2}\right)=0$, two possible situations may happen. a) The movements of any three consecutive scans from $t_{n+1}$ to $t_{n+4}$ do not satisfy either the movement or the curvature constraints (see Fig. 4(d)). b) There is a missing target point occurred at scan $t_{n+3}$.

From the above statement, a real target track should satisfy condition in Case 1 for most of the scans. Conditions in Cases 3 and 4 can happen seldom especially when missing target points are expected. By repeating this process for different $n$ at different scans and combining the results, data association can be performed. Several real examples are provided in the next section to illustrate the application of this new track identification process for clutter rejection and data association. This study involves various multitarget scenarios with different background clutter densities.

\section{SIMULATION RESULTS}

This section presents the results of the modified $\mathrm{HOC}$ for different operating conditions. These include on-line continuous detection, detection under various operating conditions and multiple target detection and track identification process.

\section{A. Continuous Mode Operation}

So far we have assumed that all the targets initiate from the first scan and remain in the field of view until the last available scan, i.e., they appear at all times. Even though this assumption was also made in many other target detection approaches, in real-life applications there is no a priori knowledge as to when the targets initiate and terminate in the sensor field of view. As a result, the detector has to operate continuously in order to be able to make decisions at any time.

The modified HOC method can easily be applied to this problem by incorporating limited memory into the process, i.e., only a limited (recent) part of the old information is kept for processing. This can be implemented fairly straightforwardly since after the available memory is full, the new incoming data can simply replace the oldest data at each scan. No extra memory is needed in the structure since the network itself records all the information that is required for processing. The memory in the HOC [1] stores the correlational information for certain number of scans. When new data arrives, the system discards the oldest data, stores the new data into the memory and then performs the decision making for the data currently available in the system. This is like a moving window process in the time domain. Therefore, detection can be made at any scan as soon as there is sufficient target information available.

To demonstrate the ability of the modified HOC process for on-line continuous operation, 16 scans of data were processed continuously and the results were reported after processing every 10-scan interval. These intervals are: $1-10,3-12,5-14$, and 7-16. The original images, with and without the clutter, 


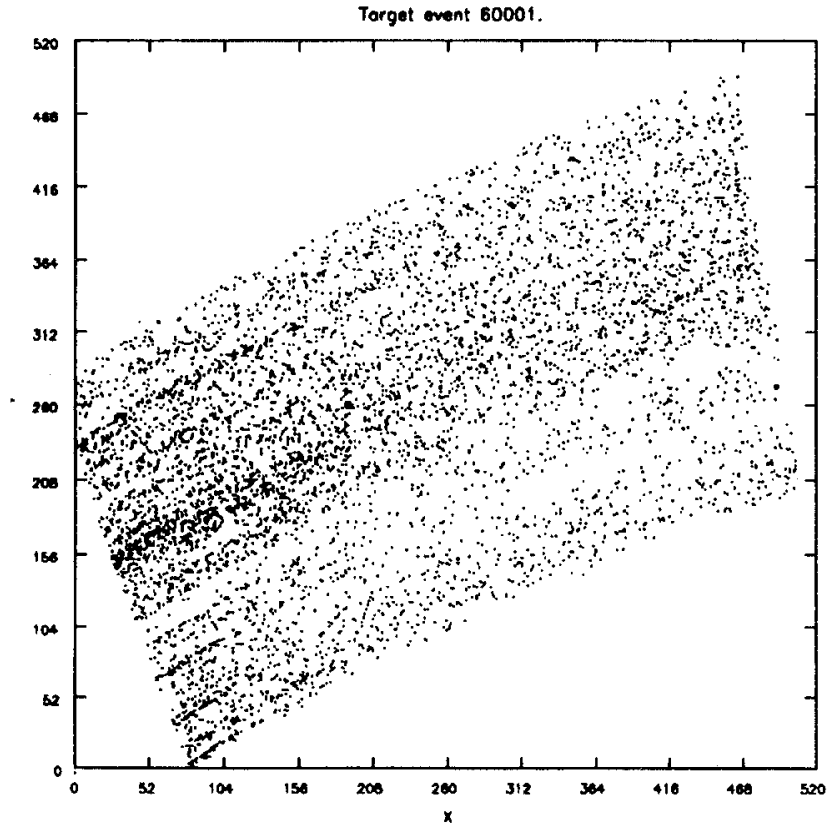

Fig. 5(a). Original cluttered image for 16 scans.

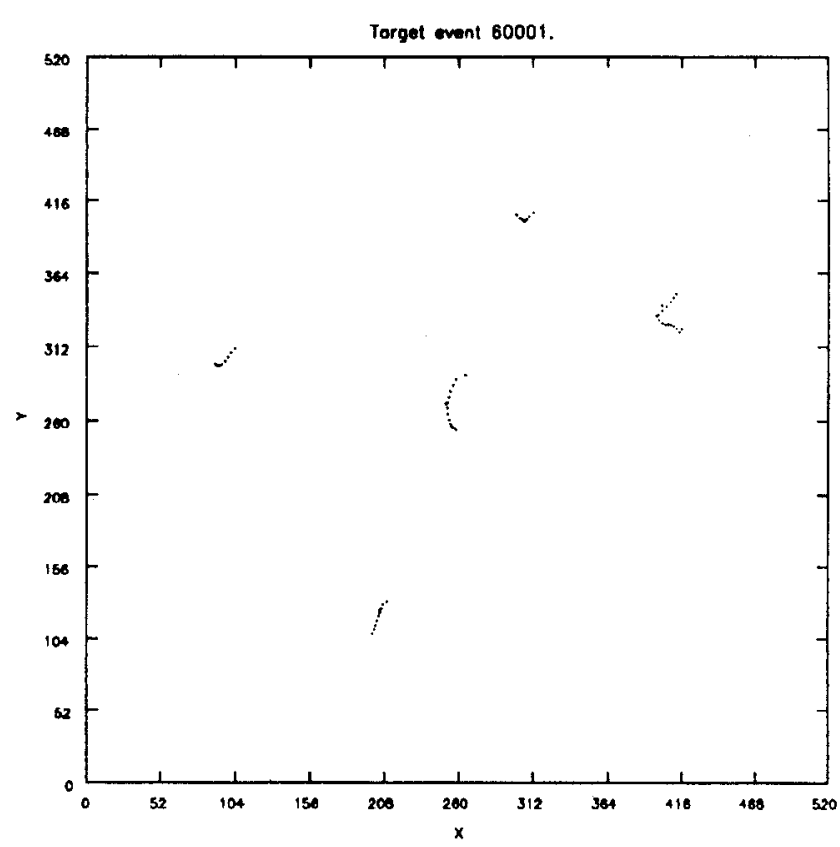

Fig. 5(b). Original clean image.

for 16 scans of data are first shown in Figs. 5(a) and 5 (b), respectively, just to show the target locations. There are six simulated target tracks in the image which are initiated and terminated at different scan numbers. These tracks are approximately located around $(100,300),(200,120),(260,270),(310,415)$, $(410,325)$, and $(420,350)$ coordinates as seen in Fig. 5(b). The first four tracks initiate at different scans within the first interval while the last two initiate within the second interval. All tracks are isolated except the last two which are splitting ones.

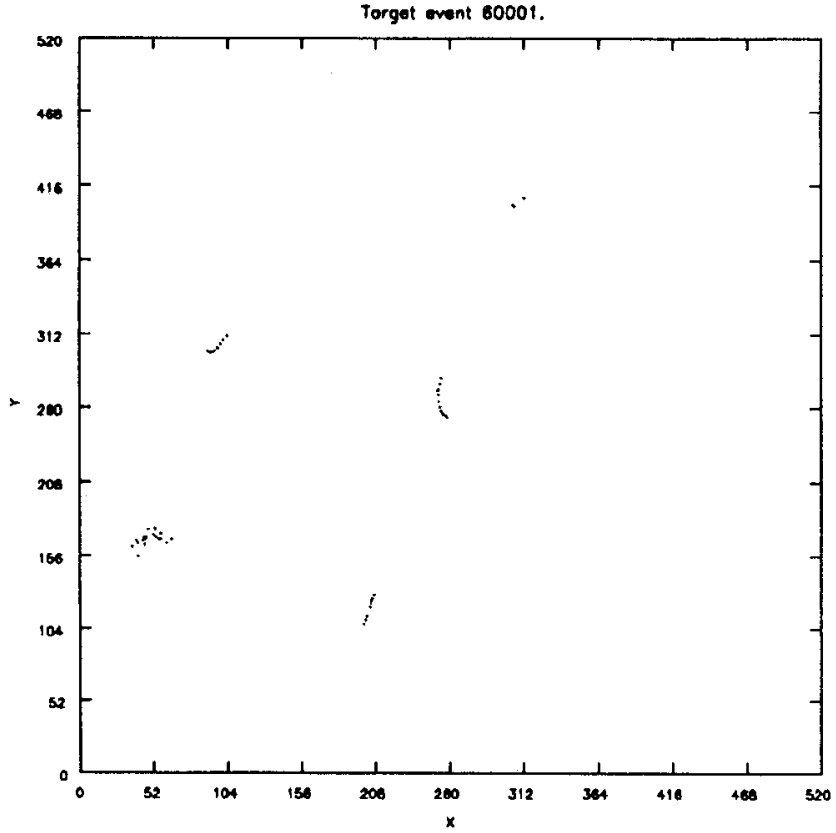

Fig. 5(c). Processed image for scans 1-10.

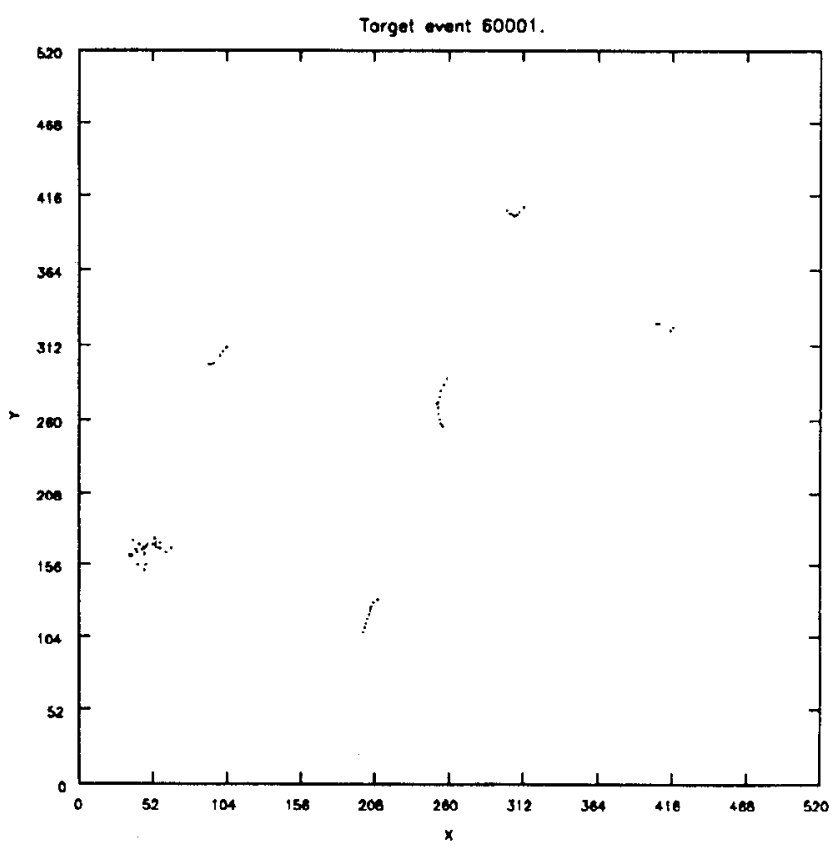

Fig. 5(d). Processed image for scans 3-12.

The processed images for the four chosen intervals are shown in Figs. 5(c), 5(d), 5(e), and 5(f), respectively. Comparing with Fig. 5(b) it is clear that all the tracks are successfully identified in their corresponding interval. The discontinuation of some of the tracks can be attributed to (1) early termination of tracks, i.e., before scan 16, and/or (2) missing target points in the relevant intervals. Note that all the points remained in the lower left part of these images correspond to stationary clutter which also satisfied the modified HOC criterion. 


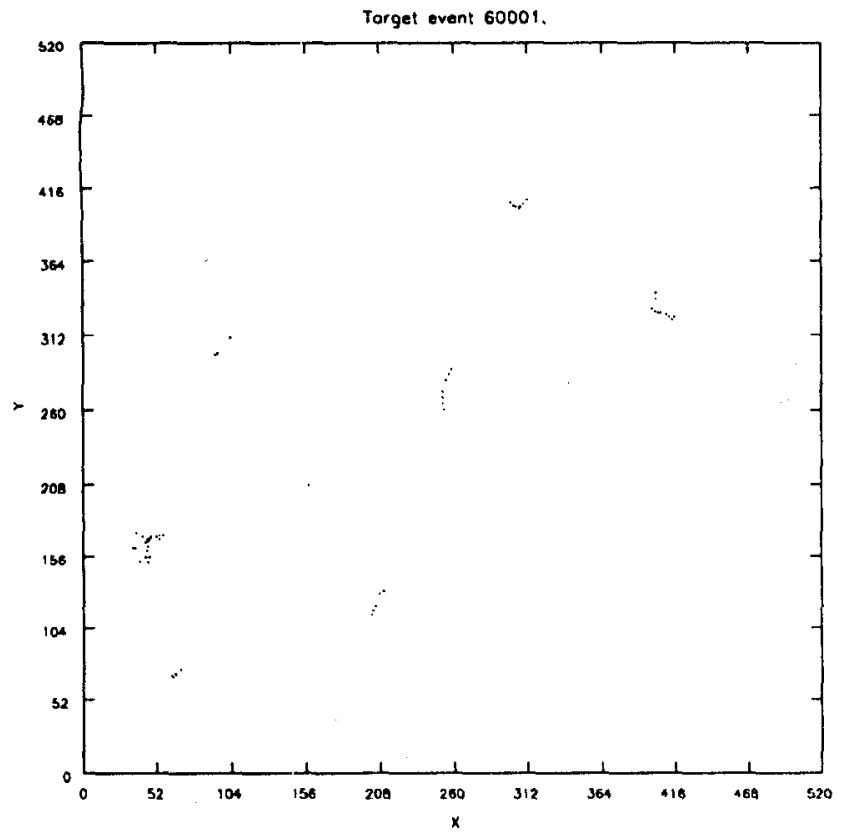

Fig. 5(e). Processed image for scans 5-11.

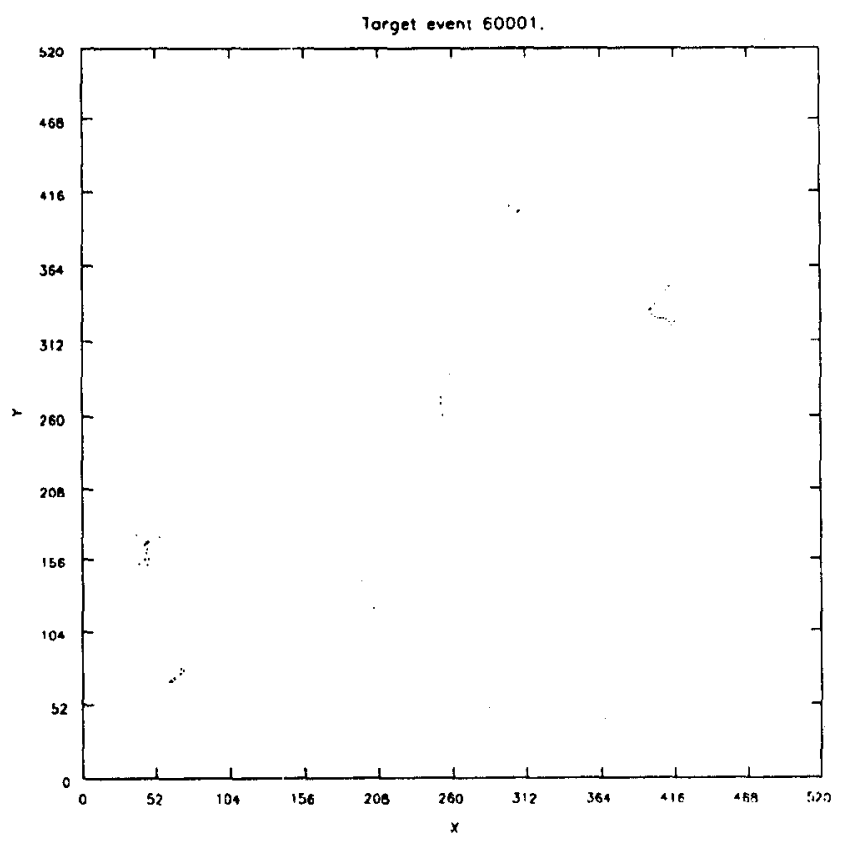

Fig. 5(f). Processed image for scans 7-16.

\section{B. Operating Characteristics}

The goal of this section is to determine the operating characteristics of the HOC method as a function of the clutter density and for different order. The upper bound on the clutter density for the HOC method is obviously dependent on the size of the movement window. The current assumption on the maximum target movement is 4 which leads to a window consisting of $(4 \times 2+1)^{2}=81$ pixels. For the original HOC method, correlation is produced when a pixel exists within the movement window

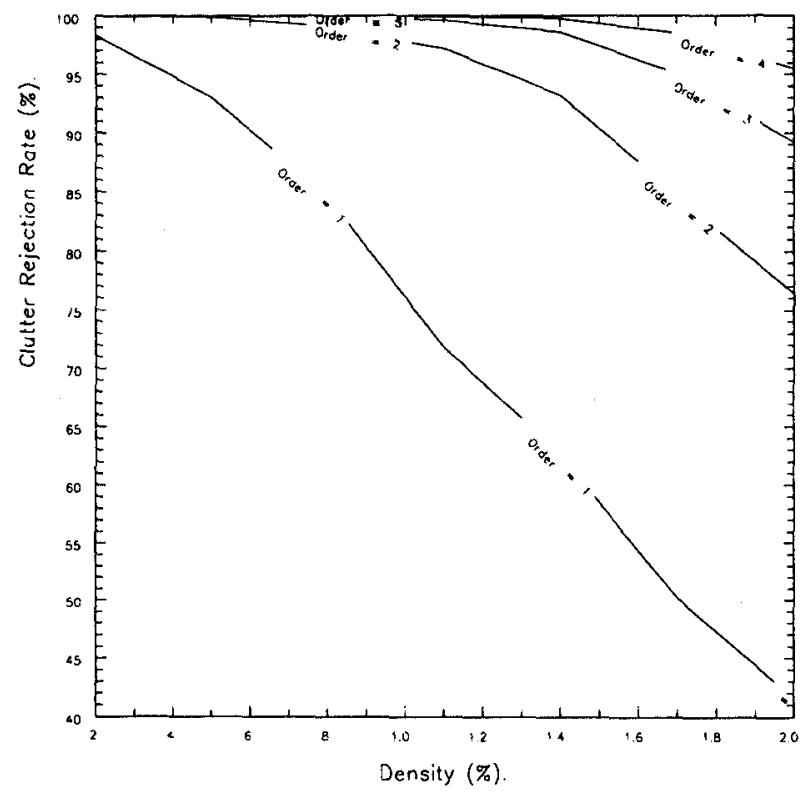

Fig. 6. Operating characteristics: fixed scan number, variable correlation number.

in the subsequent scan. If this happens continuously then consistent correlations will result. Thus, this leads to the upper bound on the clutter density for discrimination which is $1 / 81=1.23 \%$. For the modified HOC method, however, the results are dependent on the correlations between the second and the third consecutive scans. Due to the curvature constraint, the movement of the target is limited between these two scans. Thus, the allowable upper bound on the clutter density is larger in this case as the actual size of movement window is smaller. For example, if the curvature contraint is chosen to be $\delta=\pi / 4$, this window size is $1 / 4$ of the entire velocity window between the first and the second scans. Thus, in this case the upper bound on the clutter density is $(1 / 81) \times 4=4.92 \%$. Clutter densities above these limits provide consistent correlations which in turn lead to false tracks in the processed images. The above analysis may suggest that the maximum allowable clutter density can always be increased by decreasing the size of the window or reducing the curvature constraint. Even though this is true, the selected curvature constraint may not be acceptable for a particular class of targets.

To investigate the clutter rejection performance of the modified HOC method the total number of scans is assumed to be fixed while the order was varied. Ten scans of data were used for processing and the order of HOC was varied from 1 to 4 . As can be observed from the operating chracteristic plots in Fig. 6 the clutter rejection rate improves rapidly as the order of the HOC process increases. The improvement in clutter rejection is especially evident when clutter density is high. Excellent performance was achieved 
TABLE II

Clutter Rejection Results

\begin{tabular}{|c|c|c|c|c|c|}
\hline \multirow{2}{*}{ Density } & Total & \multicolumn{2}{|c|}{ MHOC results } & \multicolumn{2}{c|}{ Track identification } \\
\cline { 3 - 6 } & of points & $\begin{array}{c}\text { Points } \\
\text { remained }\end{array}$ & $\begin{array}{c}\text { Clutter } \\
\text { rejection }\end{array}$ & $\begin{array}{c}\text { Points } \\
\text { remained }\end{array}$ & $\begin{array}{c}\text { Clutter } \\
\text { rejection }\end{array}$ \\
\hline $0.8 \%$ & 13330 & 78 & $99.56 \%$ & 21 & $99.91 \%$ \\
\hline $1.1 \%$ & 18280 & 510 & $97.55 \%$ & 39 & $99.90 \%$ \\
\hline $1.4 \%$ & 23100 & 1406 & $93.99 \%$ & 29 & $99.96 \%$ \\
\hline $1.7 \%$ & 28300 & 3578 & $87.42 \%$ & 123 & $99.64 \%$ \\
\hline $2.0 \%$ & 33080 & 8139 & $75.44 \%$ & 578 & $98.31 \%$ \\
\hline
\end{tabular}

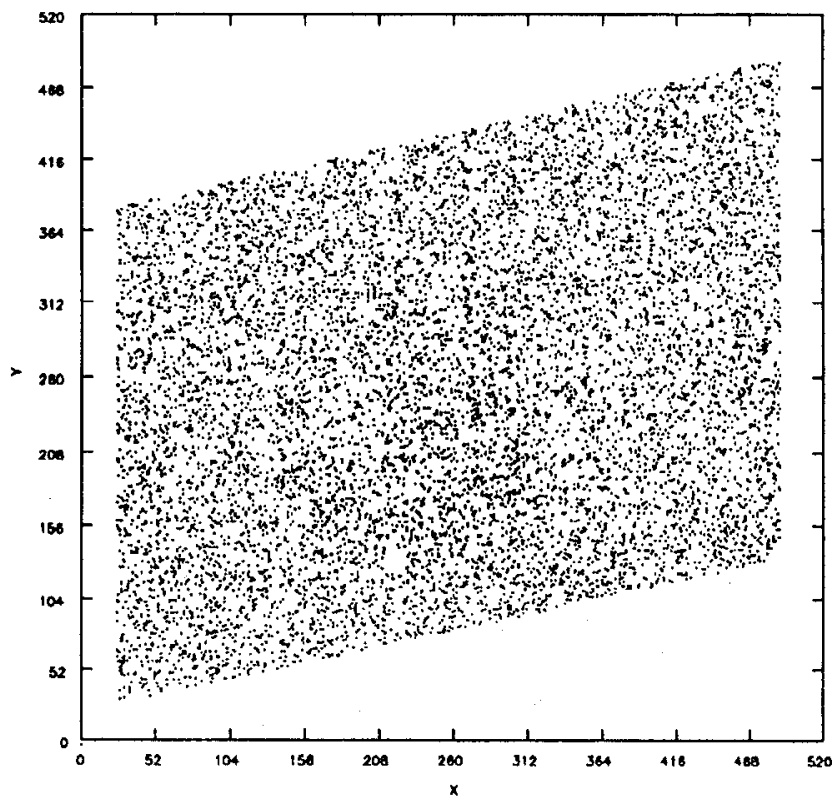

Fig. 7. Original image of clutter density $1.1 \%$.

when fourth order correlation was used. Nevertheless, the price one pays for this performance improvement is the increased chance of misdetection due to missing target points [1].

\section{Multiple Target Track Identification Results}

In this section simulations are performed on several images with different clutter densities. The tracks were identified using the procedures described in Section III and scores were calculated for each track individually by averaging all the point scores. In the scoring process, the relevant parameters in (8) and (9) were chosen to be $A_{1}=9, A_{2}=2.12$, $\alpha_{1}=1 / 3$, and $\alpha_{2}=2$. A threshold for rejecting tracks with lower scores was then chosen empirically to be 2.5. Table II shows the clutter rejection performance for different clutter densities. The first column of this table indicates the clutter densities. The numbers in the second column are the total number of points in the entire image (target as well as clutter points) before the processing. The total

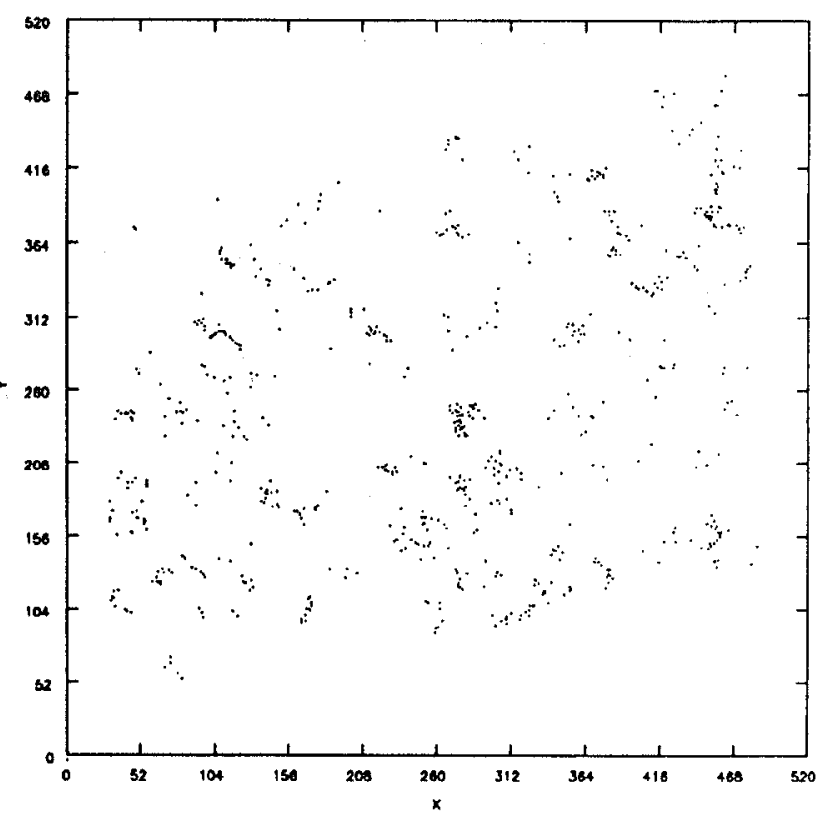

Fig. 8. Processed image of Fig. 7 after modified HOC.

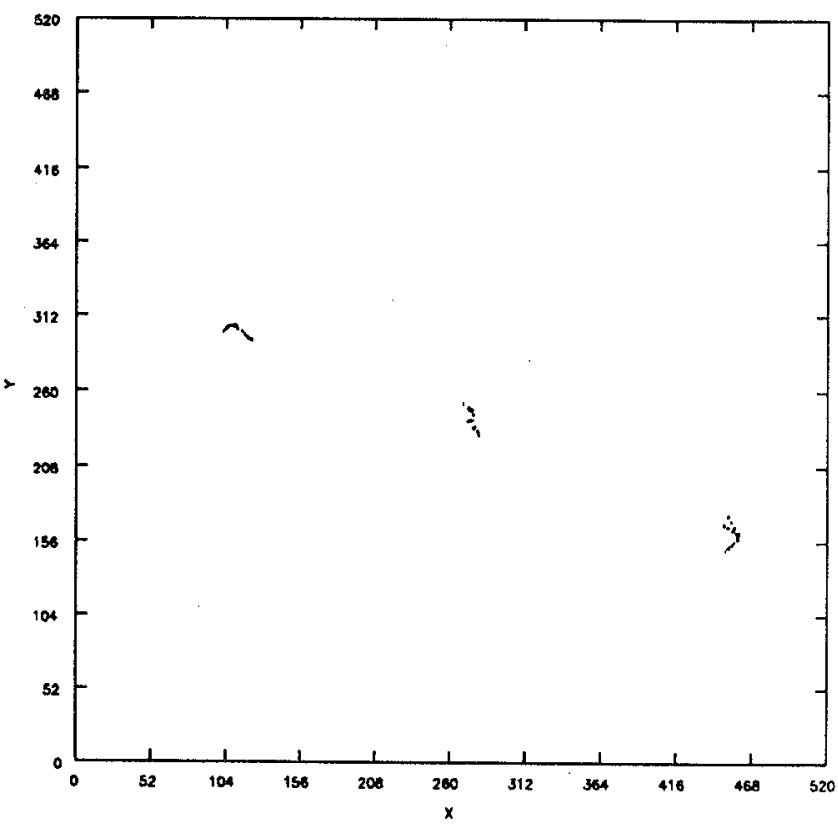

Fig. 9. Processed image of Fig. 7 using track identification.

number of points that remained in the images after the processing by the modified HOC method and the track identification process of Section III are shown in columns three and five, respectively. Since all the target information is retained, these numbers include 20 points corresponding to the two tracks each ten-point long. The corresponding clutter rejection rates are given in columns four and six, respectively. The improvement in the clutter rejection rates is clearly evident when the modified HOC is used in conjunction with the new track identification scheme. 


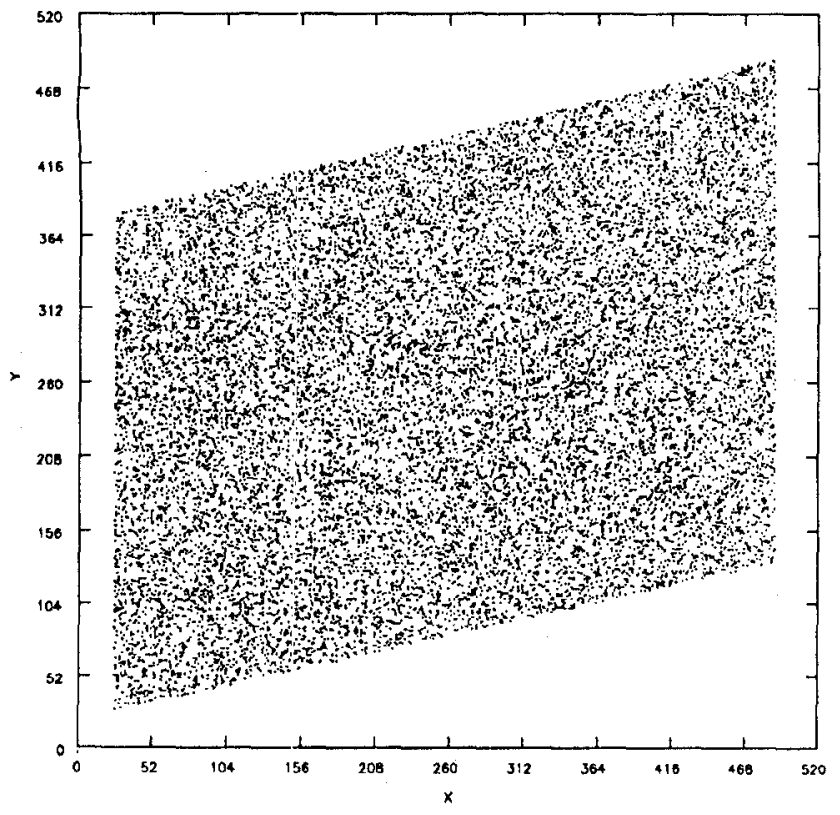

Fig. 10. Original image of clutter density $2.0 \%$.

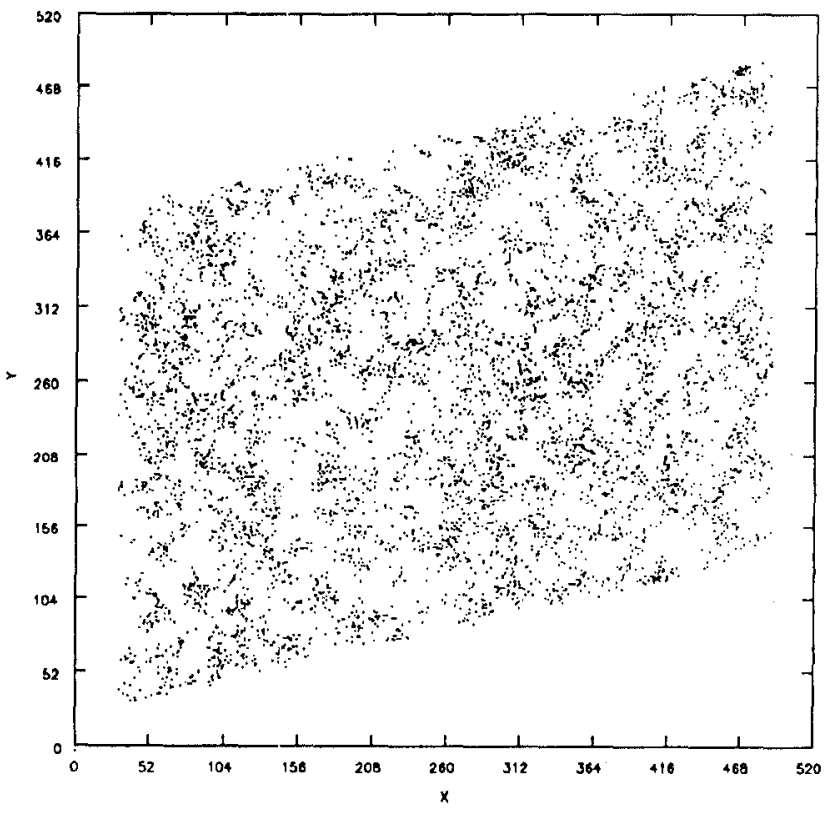

Fig. 11. Processed image of Fig. 10 after modified HOC.

The original and processed images for selected clutter densities of $1.1 \%$ and $2.0 \%$ are shown in Figs. $7-12$. Note that the clutter density is defined in terms of the number of points in the movement window as in Section IVB. There are two target tracks in these images. One is located around coordinate $(104,312)$ and the other one is located around $(468,156)$. They can clearly be seen in Fig. 9. The result in Fig. 12 indicates a large number of false tracks as the clutter density is very high in this case. Nevertheless, the significant improvements in the results of the track identification process can still be observed.

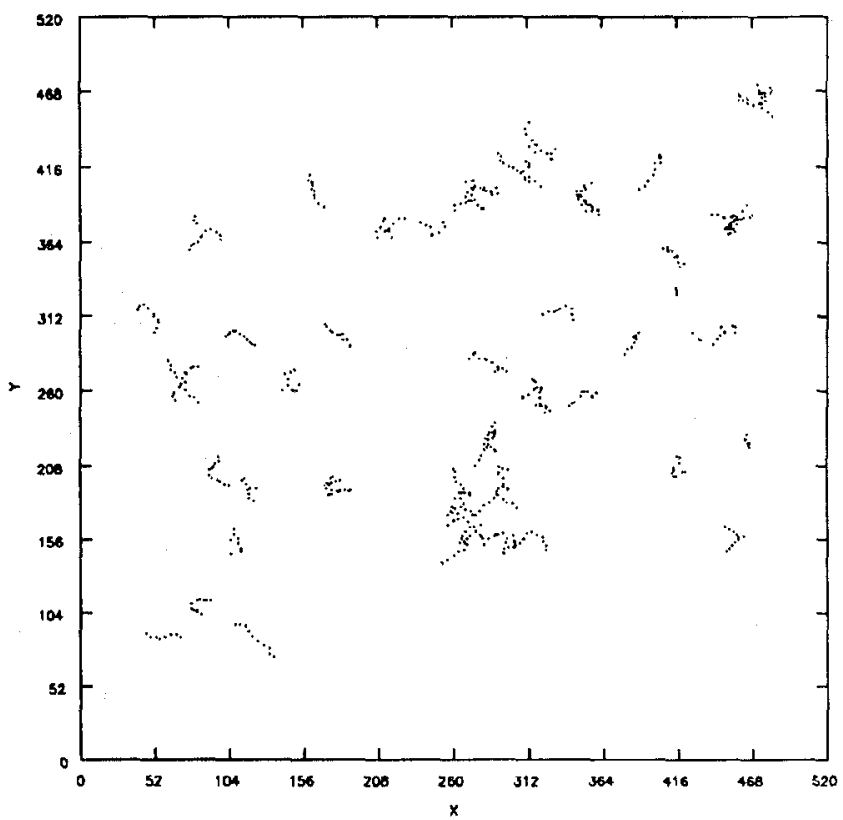

Fig. 12. Processed image of Fig. 10 using track identification.

TABLE III

Results on Multitarget Detection

\begin{tabular}{|c|c|c|c|c|c|}
\hline \multirow[b]{2}{*}{ Density } & \multirow{2}{*}{$\begin{array}{l}\text { Total } \\
\text { number } \\
\text { of points }\end{array}$} & \multicolumn{2}{|c|}{ MHOC results } & \multicolumn{2}{|c|}{ Track identification } \\
\hline & & $\begin{array}{l}\text { Points } \\
\text { remained }\end{array}$ & $\begin{array}{l}\text { Clutter } \\
\text { rejection }\end{array}$ & $\begin{array}{c}\text { Points } \\
\text { remained }\end{array}$ & $\begin{array}{l}\text { Clutter } \\
\text { rejection }\end{array}$ \\
\hline $0.8 \%$ & 319 & 39 & $96.88 \%$ & 31 & $99.66 \%$ \\
\hline $1.4 \%$ & $\overline{537}$ & 77 & $90.73 \%$ & 34 & $99.21 \%$ \\
\hline $2.0 \%$ & 738 & 181 & $78.67 \%$ & 48 & $97.46 \%$ \\
\hline
\end{tabular}

To show the usefulness of the track identification process for detection and identification of multiple close target tracks which might be crossing or splitting and also to demonstrate the potential application of this method for data association and tracking, three target tracks were synthetically created in $60 \times 60$ size images. Background clutter with three different densities was also generated. For each clutter density, six images were generated in which the target tracks were positioned and initialized randomly. This means that the relative positions of the tracks were changed by random translation and rotation operations to generate closely spaced, crossing or splitting tracks. For each event ten scans of the data were used. The original 3-D images for six events with clutter density of $1.4 \%$ are shown in Fig. 13. Both the modified HOC method and the modified HOC with track identification scheme were used and the clutter rejection results are tabulated in Table III.

The first column in this table gives the density of clutter. The second column shows the average (averaged based on six cases) total number of points in the original images for the specific density case. Columns 3 and 5, respectively, show the average number of points remained in the resultant images after the modified HOC and track identification 

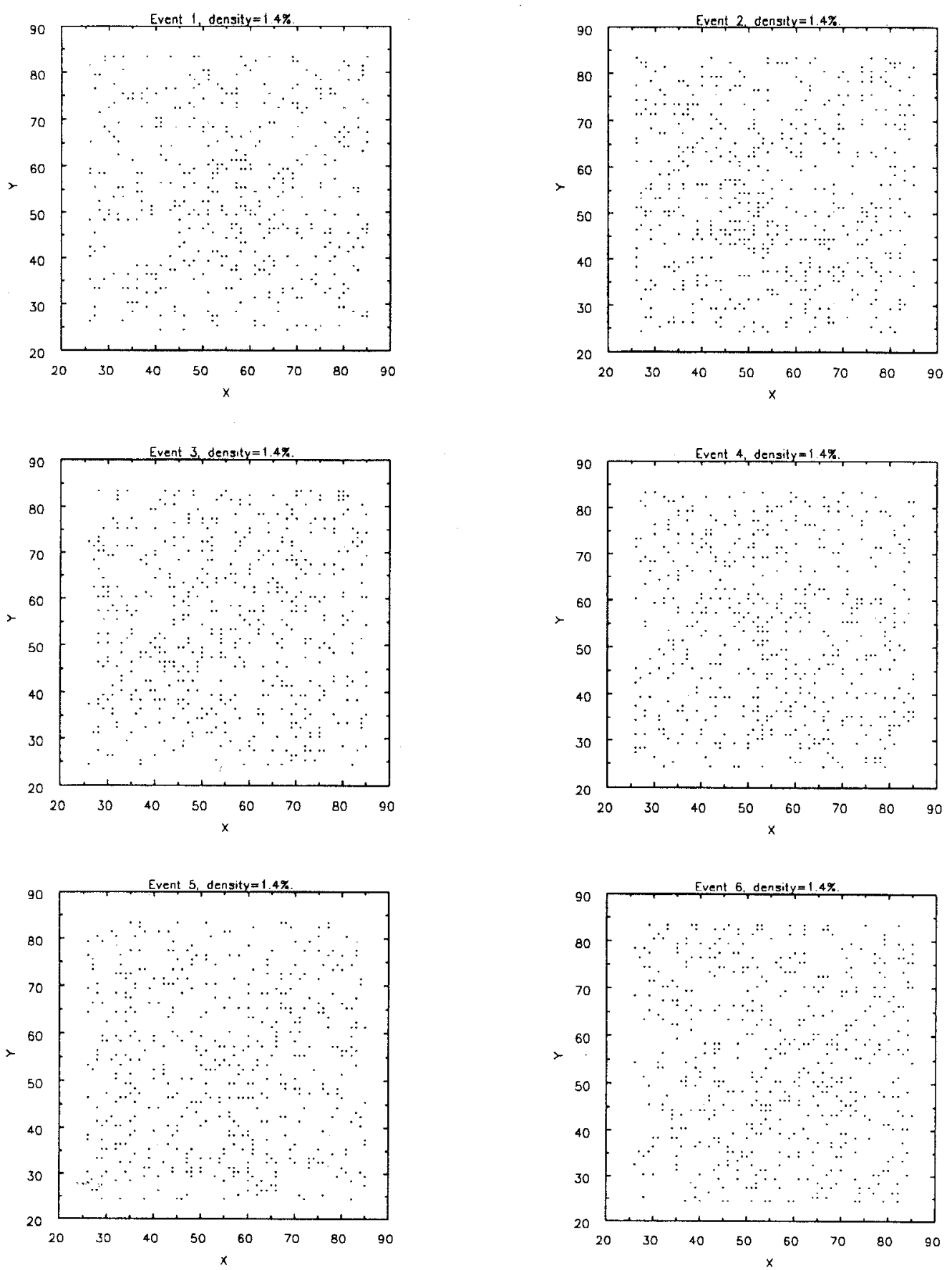

Fig. 13. Six multitarget events with density $1.4 \%$.

processes. The corresponding percentages for clutter rejection in columns 4 and 6 are arrived at taking into account that there are 3 target tracks each 10 scans long in each image. For example for the last row in the table when track identification is used the clutter rejection is $100 \times(708-18) / 708 \%=97.46 \%$. Note that no target point is lost as a result of our processing.

The actual processed images for the events with density $1.4 \%$ after track identification process are shown in Fig. 14. In these images, the associated scan numbers are marked for subsequent data association and tracking purposes. The results of track identification are significantly better than those of the modified HOC process. Using this track identification in conjunction with the track-oriented scoring process, close, splitting and/or crossing target tracks can be identified even in a very dense background clutter.

\section{CONCLUSIONS}

In this paper the modified HOC scheme was extended in order to perform track identification 

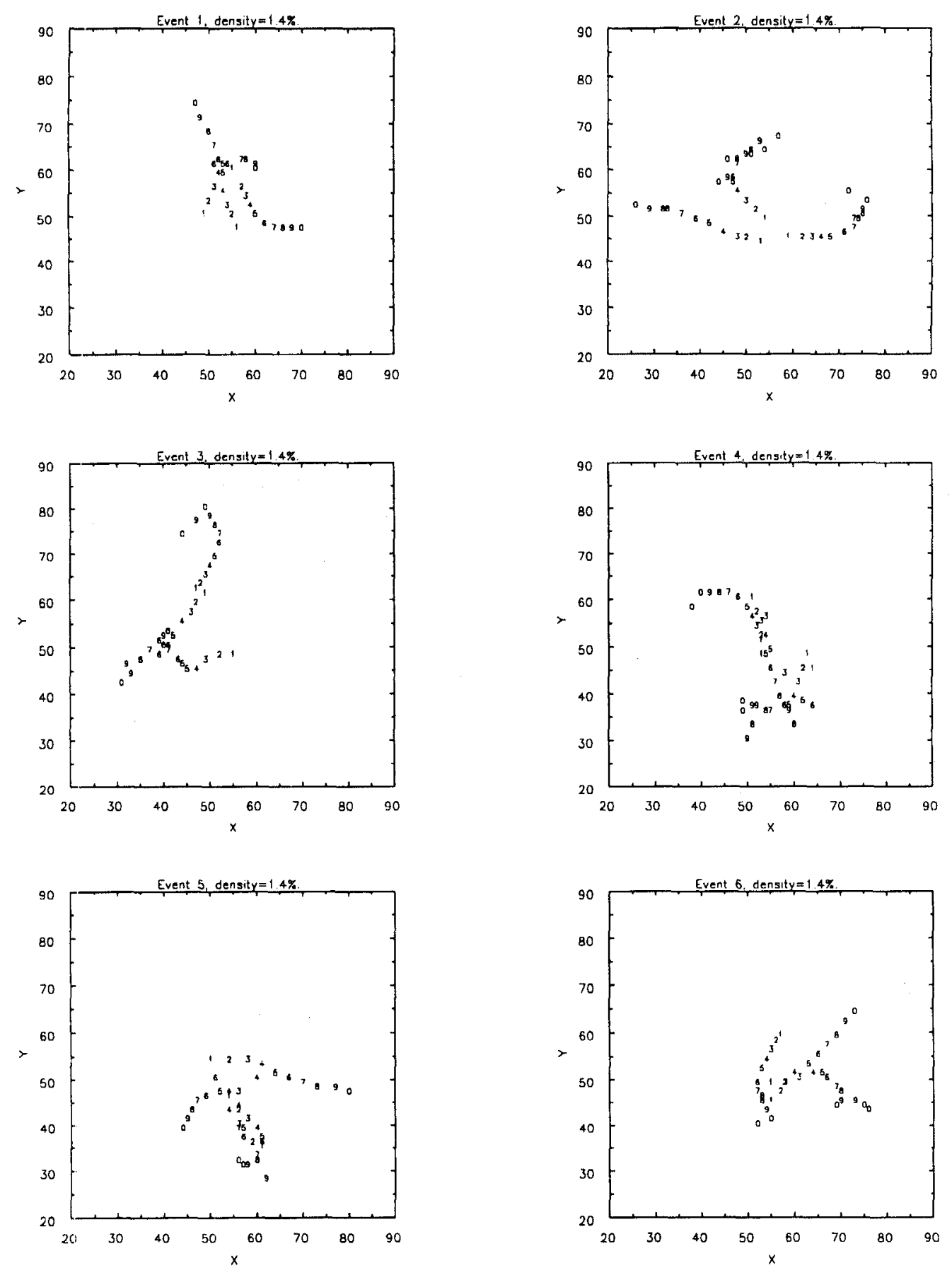

Fig. 14. Processed image of Fig. 14 after track identification.

and data association on multiple target tracks in a heavily cluttered environment. A scoring process was proposed in order to effectively discriminate target tracks with low scores particularly when the clutter density was high. A new track identification process was also developed, based on the properties of the modified HOC, which cannot only be used as an effective tool for detecting and identifying very close, crossing or splitting tracks but also can be of great use as the first step for data association and tracking purposes. The new scoring process was then used in conjunction with the track identification scheme to further improve the discrimination capability of the modified HOC method especially when multiple close, splitting and/or crossing tracks are encountered. Simulation results indicated the potential applications of the modified HOC and the new track identification processes in different operating scenarios. These include continuous mode operation for on-line implementation, operating characteristics under various clutter density and for different system parameters, and detection and identification of multiple close, splitting and/or crossing tracks in heavy clutter. In addition, it was shown that the 
modified HOC process can easily be applied to detect targets that initiate and terminate at any arbitrary scan number.

Some of the unique features of the proposed schemes in this work are as follows.

1) No a priori information pertaining to the target and/or clutter is required. These include initial conditions, target speed, number of targets in the scene, and statistics of clutter.

2) The process can detect any number of target tracks which can be very close, crossing or splitting without increasing the computational complexity of the algorithm.

3) The detection process can be performed continuously and in one step with no additional processing, e.g. track initialization.

4) The algorithm is simple in structure and can easily be implemented in a parallel processing environment.

\section{APPENDIX A}

PROOF OF PROPOSITION 1 We begin by assuming that at scan $t_{n}$, the target is located at $\left(x_{n}, y_{n}\right)$. Using (2) all the target positions $\left(x_{n}, y_{n}, t_{n}\right)$ can now be expressed in terms of the initial target location $\left(x_{1}, y_{1}\right)$ with various shifts in the spatial domain, i.e.,

$$
\begin{aligned}
& F\left(x_{2}, y_{2}, t_{2}\right)=g\left\{\sum_{i_{1}=-v}^{v} \sum_{j_{1}=-v}^{v} F\left(x_{1}+i_{1}, y_{1}+j_{1}, t_{2}\right)\right\} \\
& F\left(x_{3}, y_{3}, t_{3}\right)=g\left\{\sum_{i_{2}=-v}^{v} \sum_{j_{2}=-v}^{v} F\left(x_{2}+i_{2}, y_{2}+j_{2}, t_{3}\right)\right\} \\
&=g\left\{\sum_{i_{2}=-v}^{v} \sum_{j_{2}=-v}^{v} \sum_{i_{1}=-v}^{v} \sum_{j_{1}=-v}^{v} \ldots\right. \\
&\left.\quad F\left(x_{1}+i_{1}+i_{2}, y_{1}+j_{1}+j_{2}, t_{3}\right)\right\} \\
& \ldots \\
& \ldots \\
& \ldots \ldots \\
& F\left(x_{n}, y_{n}, t_{n}\right)=g\left\{\sum_{i_{n-1}=-v j_{n-1}=-v}^{v} \sum^{v} F\left(x_{n-1}+i_{n-1}, y_{n-1}+j_{n-1}, t_{n}\right)\right\} \\
&=g\left\{\sum_{i_{n-1}=-v j_{n-1}=-v}^{v} \sum_{i_{1}=-v}^{v} \ldots \sum_{j_{1}=-v}^{v}\right. \\
&\left.F\left(x_{1}+i_{1} \ldots+i_{n-1}, y_{1}+\cdots+j_{n-1}, t_{n}\right)\right\} .
\end{aligned}
$$

The method of induction is used in the sequel. For $k=1$, using (11) in (5) yields

$$
\begin{aligned}
Y^{(1)}\left(x_{n}, y_{n}, t_{n}\right) & \\
= & g\left[F\left(x_{n}, y_{n}, t_{n}\right) \sum_{i_{1}} \sum_{j_{1}} F\left(x_{n}+i_{1}, y_{n}+j_{1}, t_{n+1}\right)\right. \\
& \left.\quad \times \sum_{i_{2}} \sum_{j_{2}} F\left(x_{n}+i_{1}+i_{2}, y_{n}+j_{1}+j_{2}, t_{n+2}\right)\right] \\
= & F\left(x_{n}, y_{n}, t_{n}\right) F\left(x_{n+1}, y_{n+1}, t_{n+1}\right) \\
& \times F\left(x_{n+2}, y_{n+2}, t_{n+2}\right) .
\end{aligned}
$$

Note that $Y^{(0)}\left(x_{n}, y_{n}, t_{n}\right)=F\left(x_{n}, y_{n}, t_{n}\right)$. Now we assume that the result holds for order $k-1$, we will then prove that it is also valid for order $k$. Using (5) we can write

$$
\begin{aligned}
Y^{(k)}\left(x_{n}, y_{n}, t_{n}\right) & \\
=g & {\left[Y^{(k-1)}\left(x_{n}, y_{n}, t_{n}\right) \sum_{i_{n}} \sum_{j_{n}} Y^{(k-1)}\left(x_{n}+i_{n}, y_{n}+j_{n}, t_{n+1}\right)\right.} \\
& \left.\times \sum_{i_{n+1}} \sum_{j_{n+1}} Y^{(k-1)}\left(x_{n}+i_{n}+i_{n+1}, y_{n}+j_{n}+j_{n+1}, t_{n+2}\right)\right] \\
=Y^{(k-1)}\left(x_{n}, y_{n}, t_{n}\right) g & \left\{\sum_{i_{n}} \sum_{j_{n}} Y^{(k-1)}\left(x_{n}+i_{n}, y_{n}+j_{n}, t_{n+1}\right)\right. \\
& \left.\times\left[\sum_{i_{n+1}} \sum_{j_{n+1}} Y^{(k-1)}\left(x_{n}+i_{n}+i_{n+1}, y_{n}+j_{n}+j_{n+1}, t_{n+2}\right)\right]\right\} .
\end{aligned}
$$

Since it is assumed that (7) is true for order $k-1$, we have

$$
\begin{aligned}
Y^{(k-1)}\left(x_{n}, y_{n}, t_{n}\right)= & F\left(x_{n}, y_{n}, t_{n}\right) F\left(x_{n+1}, y_{n+1}, t_{n+1}\right) \\
& \times \cdots F\left(x_{n+2 k}, y_{n+2 k}, t_{n+2 k-2}\right)=1 .
\end{aligned}
$$

Also using the extension of (11) for $Y$ at different scans $t_{n}$ we obtain

$$
\begin{aligned}
& \sum_{i_{n}} \sum_{j_{n}} Y^{(k-1)}\left(x_{n}+i_{n}, y_{n}+j_{n}, t_{n+1}\right) \\
&=Y^{(k-1)}\left(x_{n+1}, y_{n+1}, t_{n+1}\right) \\
&= F\left(x_{n+1}, y_{n+1}, t_{n+1}\right) F\left(x_{n+2}, y_{n+2}, t_{n+2}\right) \\
& \times \cdots F\left(x_{n+2 k-1}, y_{n+2 k-1}, t_{n+2 k-1}\right)=1
\end{aligned}
$$

and

$$
\begin{aligned}
\sum_{i_{n}} \sum_{j_{n}} & \sum_{i_{n+1}} \sum_{j_{n+1}} Y^{(k-1)}\left(x_{n}+i_{n}+i_{n+1}, y_{n}+j_{n}+j_{n+1}, t_{n+2}\right) \\
= & Y^{(k-1)}\left(x_{n+2}, y_{n+2}, t_{n+2}\right) \\
= & F\left(x_{n+2}, y_{n+2}, t_{n+2}\right) F\left(x_{n+3}, y_{n+3}, t_{n+3}\right) \\
& \times \ldots F\left(x_{n+2 k}, y_{n+2 k}, t_{n+2 k}\right)=1 .
\end{aligned}
$$


Now, substituting these results into (12) and performing the summations and hard limiting threshold yields (7).

Q.E.D.

\section{REFERENCES}

[1] Liou, R., and Azimi-Sadjadi, M. R. (1993) Dim target delection using high order correlation method. IEEE Transactions on Aerospace and Electronic Systems, 29, 3 (July 1993), 841-856.

[2] Liou, R. J., and Azimi-Sadjadi, M. R. (1994) Multiple target detection and track identification using modified high order correlations. In Proceedings of IEEE ICNN'94, Orlando, June 1994, 3277-3282.

[3] Azimi-Sadjadi, M. R., and Liou, R. J. (1992)

Research in multiple target detection using modified high order correlation method.

Final Report to IBM Corporation, Sept. 1992.

[4] Liou, R., and Azimi-Sadjadi, M. R. (1992)

Dim target detection and clutter rejection using high order correlation method.

In Proceedings of IJCNN, Baltimore, MD, June 1992 289-294.

[5] Reed, I. S., Gagliardi, R. M., and Shao, H. M. (1983) Application of three-dimensional filtering to moving target detection.

IEEE Transactions on Aerospace and Electronic Systems, 19, 6 (Nov. 1983), 898-905.

[6] Porat, B., and Friedlander, B. (1990)

A frequency domain algorithm for multiframe detection and estimation of dim targets.

IEEE Transactions on Pattern Analysis and Machine Intelligence, 12 (Apr. 1990), 398-401.

[7] Mohanty, N. C. (1981)

Computer tracking of moving targets in space.

IEEE Transactions on Pattern Analysis and Machine Intelligence, PAMI-3, 5 (Sept. 1981), 606-611.

[8] Reid, D. B. (1979)

An algorithm for tracking multiple targets.

IEEE Transactions on Automatic Control, AC-24, 12 (Dec. 1979), 843-854.
[9] Blackman, S. S. (1986)

Multiple-Target Tracking with Radar Applications.

Dedham, MA: Artech House, 1986.

[10] Bar-Shalom, Y., and Fortman, T. E. (1988)

Tracking and Data Association.

New York: Academic Press, 1988.

[11] Bar-Shalom, Y., Shertukde, H. M., and Pattipati, K. P. (1989)

Use of measurements from an imaging sensor for precision target tracking.

IEEE Transactions on Aerospace and Electronic Systems, 25, 6 (Nov. 1989), 863-872.

[12] Bar-Shalom, Y., Shertukde, H. M., and Pattipati, K. P. (1990)

Precision target tracking for small extended objects. Optical Engineering, 29, 2 (Feb. 1990), 121-126.

[13] Shertukde, H. M., and Bar-Shalom, Y. (1991)

Tracking of crossing targets with imaging sensors.

IEEEE Transactions on Aerospace and Electronic Systems, 27, 4 (July 1991), 582-592.

[14] Shertukde, H. M., and Bar-Shalom, Y. (1990)

Detection and estimation for multiple targets with two omnidirectional sensors in the presence of false measurements.

IEEE Transactions on Acoustics, Speech, and Signal Processing, 38, 5 (May 1990), 749-763.

[15] Roth, M. (1989)

Neural networks for extraction of weak targets in high clutter environments.

IEEE Transactions on Systems, Man, and Cybernetics, 19, 10 (Oct. 1989), 1210-1217.

[16] Lee Giles, C., and Maxwell, T. (1987)

Learning, invariance, and generalization in high-order neural networks.

Applied Optics, 26, 23 (Dec. 1987), 4972-4978.

[17] Rumelhart, D. E., McClelland, J. L., and PDP Research Group (1986)

Parallel Distributed Processing.

Cambridge, MA: MIT Press, 1986. 

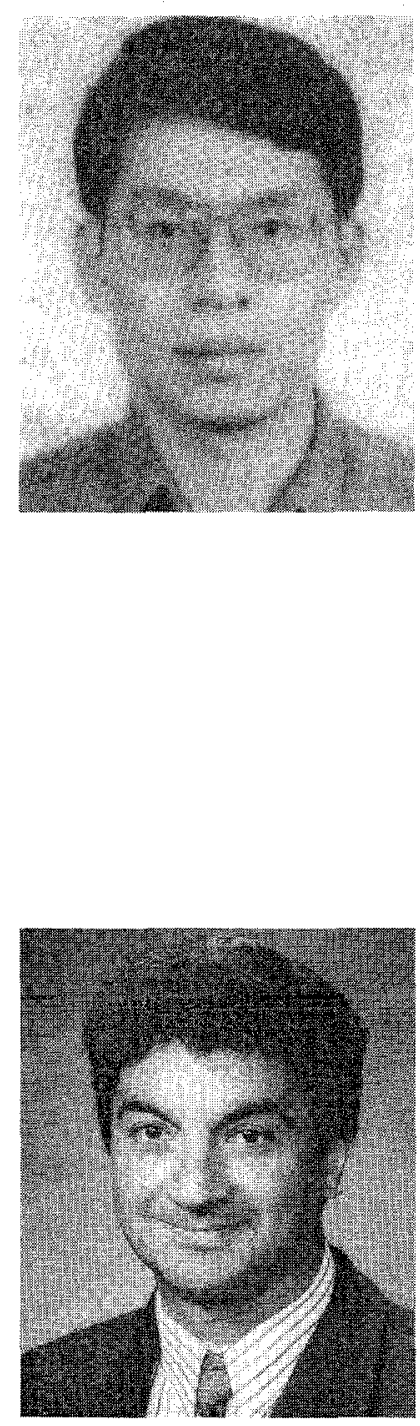

Ren-Jean Liou (S'87-M'93) received a diploma in electrical engineering from Ming-Chi Institute of Technology, Taipei, Taiwan, ROC, in 1984; the M.S. degree in electrical engineering from Marquette University, Milwaukee, WI, in 1989; and the Ph.D. degree in electrical engineering from Colorado State University, Ft. Collins, CO, in 1993.

He was a visiting scientist at Cooperative Institute for Research in the Atmosphere, Colorado, from 1993-1994. Since February 1994, he has been with the Department of Electrical Engineering at Da-Yeh Institute of Technology, Changhua, Taiwan, ROC, where he is currently an Associate Professor. His research interests are in the areas of digital signal/image processing and neural networks.

Mahmood R. Azimi-Sadjadi (S'81-M'81-SM'89) was born in Tehran, Iran in 1952. He received the B.S. degree from University of Tehran, Iran in 1977, and the M.Sc. and Ph.D. degrees from the Imperial College, University of London, England, in 1978 and 1982, respectively, all in electrical engineering.

He served as an Assistant Professor in the Department of Electrical and Computer Engineering, University of Michigan-Dearborn. Since July 1986 he has been with the Department of Electrical Engineering, Colorado State University, where he is now a Professor. He is also the director of the Multi-Sensory Computing Laboratory (MUSCL) at Colorado State University. His areas of interest are digital signal/image processing, target detection and tracking, multidimensional system theory and analysis, adaptive filtering, system identification and neural networks.

Dr. Azimi-Sadjadi has published in over 100 journals and refereed conference publications. Dr. Azimi-Sadjadi is the co-author of the book, Digital Filtering in One and Two Dimensions, Plenum Press, 1989. He is the recipient of 1993 ASEE-Navy Senior Faculty Fellowship Award, 1991 CSU Dean's Council Award, 1990 Battelle Summer Faculty Fellowship Award, and the 1984 DOW Chemical Outstanding Young Faculty Award of the American Society for Engineering Education. $\mathrm{He}$ is an Associate Editor of the IEEE Transactions on Signal Processing. 